\title{
IL4RA on lymphatic endothelial cells promotes $T$ cell egress during sclerodermatous graft versus host disease
}

\author{
Katia Urso, ${ }^{1}$ David Alvarez, ${ }^{2}$ Viviana Cremasco, ${ }^{3}$ Kelly Tsang, ${ }^{1}$ Angelo Grauel, ${ }^{3}$ Robert Lafyatis, ${ }^{4}$ \\ Ulrich H. von Andrian, ${ }^{2,5}$ Joerg Ermann, ${ }^{1}$ and Antonios O. Aliprantis ${ }^{1}$ \\ 'Department of Medicine, Division of Rheumatology, Immunology and Allergy, Brigham and Women's Hospital and \\ Harvard Medical School, ${ }^{2}$ Department of Microbiology and Immunobiology, Harvard Medical School, ${ }^{3}$ Department of Cancer \\ Immunology and Virology, Dana-Farber Cancer Institute, "Department of Medicine, Boston University School of Medicine, \\ Boston, Massachusetts, USA. ${ }^{5}$ Ragon Institute of Massachusetts General Hospital (MCH), MIT, and Harvard, Cambridge, \\ Massachusetts, USA.
}

Systemic sclerosis (SSc) is a potentially fatal autoimmune disorder with limited therapeutic options. Sclerodermatous graft versus host disease (sclGvHD), induced by transfer of B10.D2 splenocytes into BALB/c Rag2 $^{-/-}$mice, models an inflammatory subset of SSc characterized by a prominent IL13induced gene expression signature in the skin. Host mice deficient in IL4RA, a subunit of the type II IL4/IL13 receptor, are protected from scIGvHD. While IL4RA has a well-established role in Th2 differentiation and alternative macrophage activation, we report here a previously unappreciated function for IL4RA in lymphatic endothelial cells (LECs): regulation of activated T cell egress. Seven days after splenocyte transfer, $/ 14 \mathrm{ra}^{-/-}$hosts had increased numbers of activated graft CD4 ${ }^{+}$ T cells in skin draining lymph nodes (dLNs) but fewer T cells in efferent lymph, blood, and skin. Sphingosine-1 phosphate (S1P), master regulator of lymphocyte egress from LNs, was lower in dLNs of $/ 14 \mathrm{ra}^{-/-}$hosts with a corresponding decrease of S1P kinase 1 (Sphk1) expression in LECs. Bypassing the efferent lymphatics via i.v. injection of CD4+ $\mathrm{T}$ cells from dLNs of $/ 14 \mathrm{ra}^{-/-}$scIGvHD mice restored clinical GvHD in secondary $114 \mathrm{ra}^{-/-}$recipients. These results identify a role for IL4RA and suggest that modulation of lymphocyte egress from LNs may be effective in SSc and GvHD.

Conflict of interest: The authors declare that no conflict of interest exists.

Submitted: April 13, 2016 Accepted: July 7, 2016 Published: August 4, 2016

Reference information: JCI Insight. 2016;1(12):e88057. doi:10.1172/jici.insight.88057.

\section{Introduction}

Systemic sclerosis (SSc) is an autoimmune disease characterized by inflammation and fibrosis in skin and internal organs. This aggressive disease has the highest mortality rate among all rheumatic diseases (1), but patient heterogeneity and limited insight into pathophysiology have hindered efforts to find effective treatments (2).

Gene expression-profiling studies in lesional skin have identified several molecular subsets of SSc. Most patients with diffuse cutaneous SSc, the most severe form of the disease, fall within 2 groups, diffuse-proliferative and inflammatory, which display upregulation of cell cycle and inflammation-related genes, respectively (3). The sclGvHD mouse model, induced by adoptive transfer of B10.D2 splenocytes into BALB/c

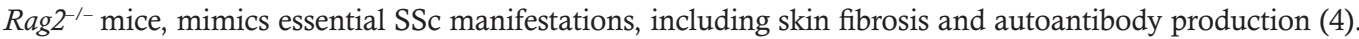
We recently demonstrated that the gene expression profile of affected skin in sclGvHD mice strongly resembles the inflammatory subset of SSc, including a prominent signature of IL13-induced genes $(5,6)$.

IL13, along with IL4, activates the type 2 differentiation program in adaptive (i.e., Th2 cells) and innate immune cells (e.g., M2 macrophages, ILC2, mast cells) (7). IL4-receptor $\alpha$ (IL4RA) is the key component of the IL4 and IL13 receptors. In hematopoietic cells, a heterodimer of IL4RA with the common $\gamma$-chain is known as the type I IL4 receptor (IL4R-I) and specifically binds IL4. In B lymphocytes, myeloid cells, and stromal cells, IL4RA also forms a complex with IL13RA1, generating the type II IL4 receptor (IL4RII), which binds both IL4 and IL13 $(8,9)$. Consistent with a functional role of IL13-induced genes in the pathogenesis of the sclGvHD model, we found that IL4RA-deficient host mice were resistant to sclGvHD and failed to develop alopecia and high-grade fibrosis (5). However, the precise role of IL4RA in the development of sclGvHD remained unresolved. 
Here, we report an essential function for IL4RA in skin draining lymph nodes (dLNs) of mice with sclGvHD. Il4ra ${ }^{-/-}$hosts accumulate more activated graft $\mathrm{T}$ cells in dLNs, and fewer of these cells are detectable in the efferent lymph, blood, and skin compared with controls. Mechanistically, IL4RA appears to control the expression of Sphk1 in LECs during the early phase of sclGvHD. Thus, in the absence of IL4RA, sphingosin-1 phosphate (S1P) levels are reduced in the efferent lymphatics and effector T cells are trapped in dLNs. Taken together, our results identify a role for IL4RA on LECs as a key factor for S1P regulation and for lymphocyte egress, a checkpoint that could be leveraged to control the progression of autoimmune diseases like SSc.

\section{Results}

IL4RA-deficient hosts are protected from sclGvHD. Alopecia is a major clinical feature in the sclGvHD model

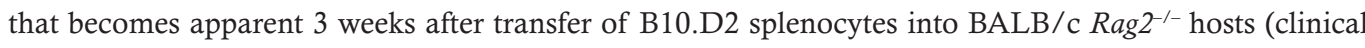
score 2 = alopecia involving $<25 \%$ of body surface) progressing over the next weeks to involve more body surface (clinical score $3=$ alopecia involving $>25 \%$ of body surface) $(4,5)$. Host mice lacking IL4RA (sclGvHD $\mathrm{Il}_{4 \mathrm{ra}^{-/-}}$mice) fail to develop clinical signs of sclGvHD skin disease (Figure 1A) (5). Searching for mechanisms contributing to protection in these mice, we examined sclGvHD $\mathrm{Il4ra}^{+/+}$and sclGvHD $\mathrm{Il}_{4 \mathrm{ra}^{-/-}}$hosts on day 7 after splenocyte transfer (referred to hereafter as 7d-sclGvHD and 7d-sclGvHD $\mathrm{Il}_{4 \mathrm{ra}^{-/-}}$mice, respectively). At this time point, sclGvHD in $\mathrm{Il}_{4 \mathrm{ra}}{ }^{+/+}$mice is characterized by weight loss and histological skin inflammation, while clinical signs of alopecia have not yet developed $(4,5)$. Compared with 7d-sclGvHD mice, 7d-sclGvHD $\mathrm{Il}_{4 \mathrm{ra}^{-/}}$mice had lost $50 \%$ less body weight (Figure $1 \mathrm{~B}$ ), and histological skin inflammation was significantly reduced (Figure 1, C and D) with a significantly lower number of skin-infiltrating $\mathrm{CD}^{+} \mathrm{T}$ cells (Figure $1, \mathrm{E}$ and $\mathrm{F}$ ). These data demonstrate that IL4RA expression by host cells critically affects the early events in sclGvHD.

Host IL4RA deficiency does not impair alloactivation of graft T cells in skin dLNs. We hypothesized that skin inflammation in 7d-sclGvHD $I 4 \mathrm{ra}^{-/-}$hosts might be reduced because of defective priming of alloreactive graft $\mathrm{T}$ cells in skin dLNs (10). However, the frequency of activated $\mathrm{CD} 4^{+} \mathrm{CD} 69^{+} \mathrm{T}$ cells was similar in the dLNs of 7d-sclGvHD and 7d-sclGvHD $I_{4 r^{-/-}}$hosts (Figure 2A), while the proportion of ki67+ proliferating $\mathrm{T}$ cells was marginally higher in $7 \mathrm{~d}-\mathrm{sclGvHD} \mathrm{Il}_{4 \mathrm{ra}^{-/}}$mice (Figure $2 \mathrm{~B}$ ). There was also no difference in the frequency of effector-memory $\mathrm{T}$ cells $\left(\mathrm{T}_{\mathrm{EM}}\right.$ cells, $\mathrm{CD} 44^{+} \mathrm{CD} 62 \mathrm{~L}^{-}$) (Figure $2 \mathrm{C}$ ), the key $\mathrm{T}$ cell population in GvHD that migrates from dLNs to the skin to propagate disease (11), suggesting that the priming of alloreactive graft $\mathrm{T}$ cells in $7 \mathrm{~d}-\mathrm{sclGvHD} \mathrm{Il}_{4 \mathrm{ra}^{-/}}$mice was not impaired. In fact, contrary to our expectation, we found that the total cell count and the number of $C D 4^{+} \mathrm{T}$ cells were significantly higher in the dLNs of 7d-sclGvHD Il4ra ${ }^{-1}$ compared with 7d-sclGvHD mice (Figure 2, D and E). Consistent with this increase in cellularity, dLN weights were also significantly higher in 7d-sclGvHD $\mathrm{Il}_{\mathrm{rra}}{ }^{-/-}$mice (Figure 2F), whereas there was no difference in spleen weights (Supplemental Figure 1; supplemental material available online with this article; doi:10.1172/jci.insight.88057DS1).

Egress of activated alloreactive T cells from dLNs is impaired in IL4ra ${ }^{-/-}$hosts. One potential explanation for finding more T cells in dLNs and fewer T cells in the skin of 7d-sclGvHD Il4ra ${ }^{-/-}$mice compared with controls would be a defect in lymphocyte trafficking in $\mathrm{IlAra}^{-/-}$hosts, in particular a defect in lymphocyte egress from the dLNs. To test this hypothesis, we measured T cell numbers in lymph and blood of 7d-sclGvHD and 7d-sclGvHD Il4ra ${ }^{-1}$ mice. Lymph collected by thoracic duct cannulation from 7d-sclGvHD Il4ra ${ }^{-1-}$ mice contained fewer total cells, mostly due to a marked reduction in $\mathrm{CD} 4^{+} \mathrm{T}$ cells, which represent about $80 \%$ of all cells in the lymph of $7 \mathrm{~d}$-sclGvHD mice (Figure $3, \mathrm{~A}-\mathrm{C}$ ). The number of total and $\mathrm{CD}^{+} \mathrm{T}$ cells in the blood of 7d-sclGvHD Il4ra ${ }^{-1}$ mice was also significantly reduced compared with 7d-sclGvHD mice (Figure 3, D-F). We first considered that the apparent defect in T cell egress from the dLNs in sclGvHD $\mathrm{Il}_{4 \mathrm{ra}^{-1}}$ mice might be caused by upregulation of retention signals such as CCL19 and CCL21, the ligands of CCR7 (12). However, expression of $C c l 19$ and $C c l 21$ measured in whole dLN cDNA preparations by quantitative PCR (qPCR) was lower in 7d-sclGvHD Il4ra ${ }^{-1}$ dLNs compared with dLNs from 7d-sclGvHD controls (Figure 3, G and H), implying that the increased cellularity of 7d-sclGvHD Il4ra ${ }^{-/}$dLNs was not due to upregulation of CCL19 or CCL21 (13).

S1P production is impaired in $\mathrm{dLN}$ of $\mathrm{Il}_{\mathrm{rra}} \mathrm{F}^{-/}$hosts. T cell egress from lymph nodes is controlled by S1P signals. Stromal cells secrete S1P, generating a gradient with increasing extracellular S1P levels in the direction of the efferent lymphatic vessels. Early after activation, T cells express low surface levels of the S1P receptor S1P receptor 1 (S1PR1). After several rounds of proliferation, expression of S1PR1 on the T cell 
A $\rightarrow$ sclGvHD $\rightarrow$ sclGvHD $\| 4 r a^{-}$
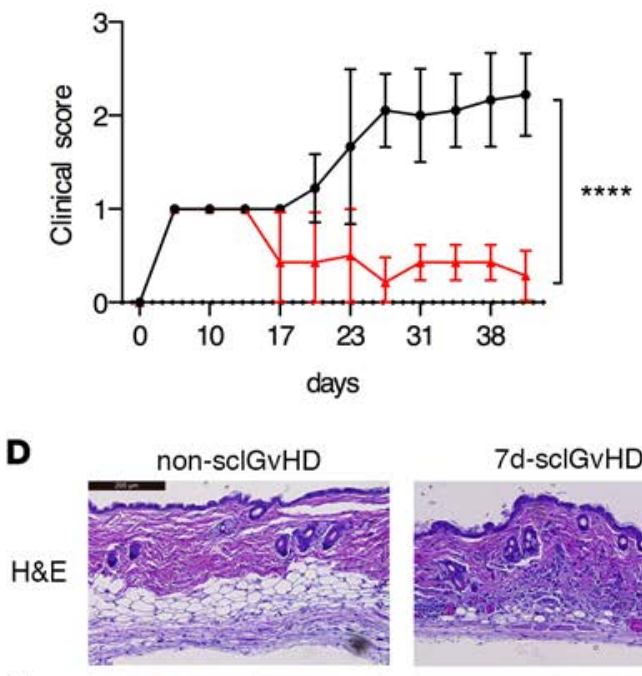

E

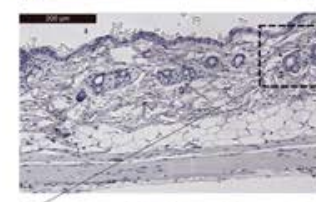

IHC

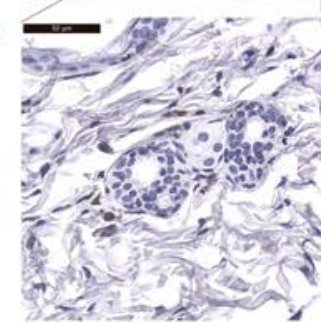

B

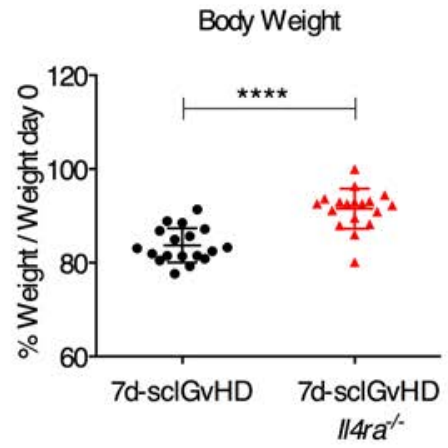

C

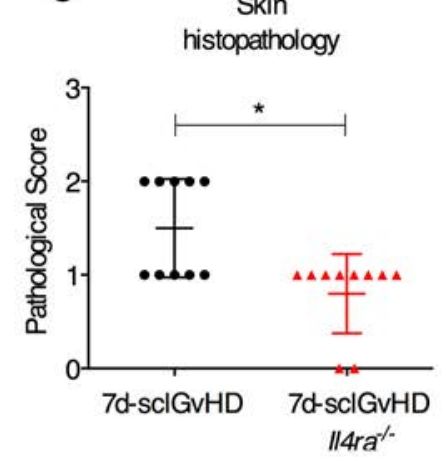

Figure 1. IL4RA-deficient hosts are protected from scIGvHD. (A) Clinical scores of sclGvHD and sclGvHD $/ / 4 \mathrm{ra}^{-/-}$mice observed for 6 weeks ( $n=7-9$ pe group, ${ }^{* * *} P<0.0001,2$-way ANOVA with Bonferroni post-test). (B) Body weight (\% of baseline) 7 days after splenocyte transfer ( $n=18$ per group; ${ }^{* * * *} P<0.0001$, Student's $t$ test). (C and D) Pathological score of inflammation (C) based on H\&E sections (D) of back skin from 7d-sclGvHD and 7d-sclGvHD $/ / 4 \mathrm{ra}^{-/-}$mice ( $n=10$ per group; ${ }^{*} P<0.05$, Mann-Whitney $U$ test). (D and E) Representative (D) H\&E staining and (E) CD3 immunostaining of back skin sections from 7d-sclGvHD and 7d-sclGvHD //4ra-/- mice; 10x. Scale bar: $200 \mu \mathrm{m}$ (D and E [upper panel]); $40 \times$. Scale bar: $50 \mu \mathrm{m}$ (E [lower panel]). (F) Quantification of $\mathrm{CD}^{+}$cells in skin sections ( $n=9-10$ per group, 4 fields/mouse (20x pictures) were evaluated, **** $P<0.0001$, Student's $t$ test). All graphs represent mean and \pm SD. The graphs represent data pooled from 3 independent experiments. The experiment shown in $\mathbf{A}$ are data pooled from 2 independent experiments to confirm the previous data published in ref. 5.

surface is restored, and the S1P concentration gradient guides the T cells out of the dLN into the efferent lymphatics, allowing them to reach the blood circulation and ultimately their target organs (11). A superagonist of the S1PR1 receptor, FTY720, has been shown to inhibit the S1P pathway, resulting in lymphopenia in lymph and blood $(14,15)$. When we treated sclGvHD mice with FTY720 for 1 week after splenocyte transfer, we observed enhanced accumulation of graft $\mathrm{T}$ cells in the dLNs and reduced $\mathrm{T}$ cell infiltration in the skin (Figure 4, A and B), similar to the phenotype of sclGvHD $I 14 \mathrm{ra}^{-1-}$ mice.

Using a previously described bioassay $(16,17)$, we measured S1P in cell-free extracts of dLNs (LN media) from 7d-sclGvHD and 7d-sclGvHD $\mathrm{Il4ra}^{-/-}$mice. LN media from 7d-sclGvHD Il4ra ${ }^{-/-}$mice contained significantly less S1P than 7d-sclGvHD controls (Figure 4C). As the concentration of S1P in lymph nodes is generally low except for the medulla $(14,18)$, the lower S1P concentration in LN media isolated from 7d-sclGvHD $I l 4 r a^{-1-}$ mice is indicative of a weaker S1P concentration gradient in these mice. There was no difference in S1PR1 surface expression on CD4 $4^{+} \mathrm{T}$ cells from the dLNs of $\mathrm{Il}_{\mathrm{ra}}{ }^{+/+}$ and IL4ra ${ }^{-/-}$hosts (Figure 4D).

Extracellular S1P levels are controlled by the kinases SPHK1 and SPHK2, which generate S1P from membrane-derived sphingosine, the transporter spinster homolog 2 (SPNS2) that mediates S1P secretion 
dLNs

A Live gate, $\mathrm{CD}^{+}$

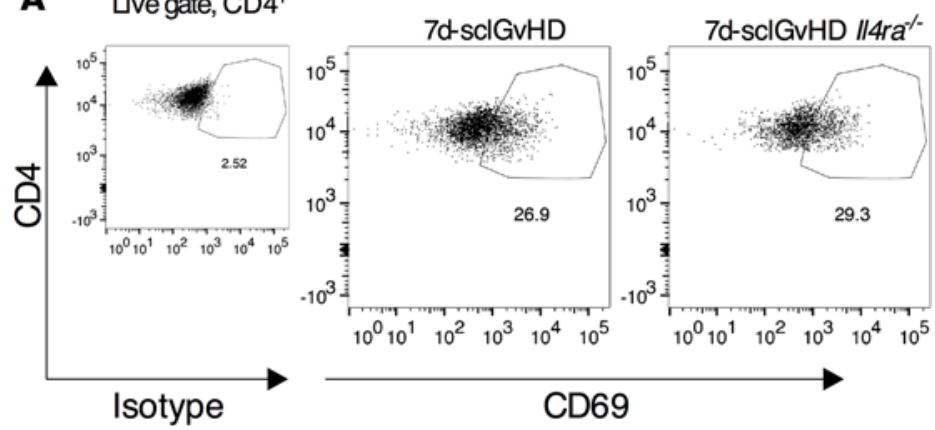

dLNs

B Live gate, $\mathrm{CD}^{+}$

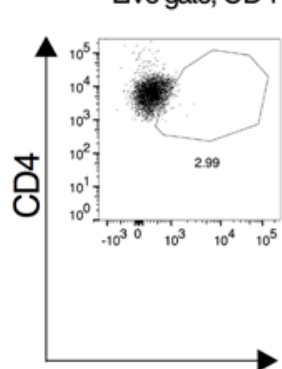

Isotype

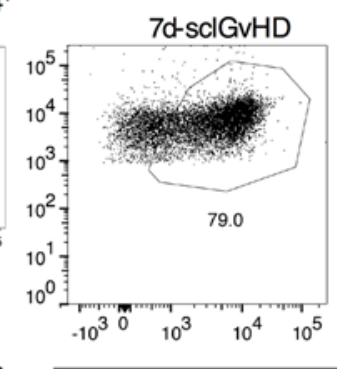

ki67

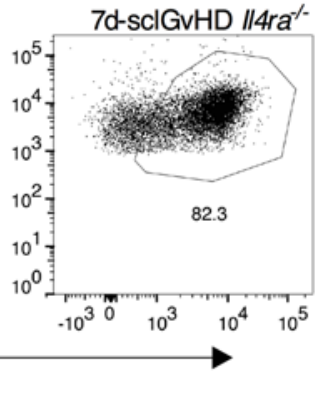

dLNs

$\mathrm{CD}^{+}{ }^{+} \mathrm{T}$ cells

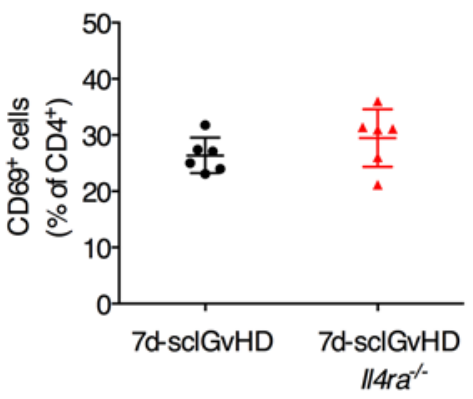

ki67 ${ }^{+} \mathrm{T}$ cells

$\begin{array}{ll}\text { C } & \text { LLNs } \\ \text { Live gate, } C D 4^{+}\end{array}$
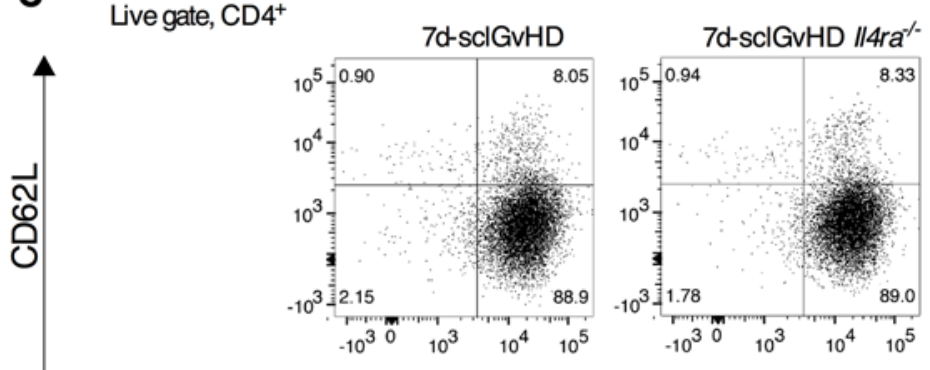

CD44

dLN

D

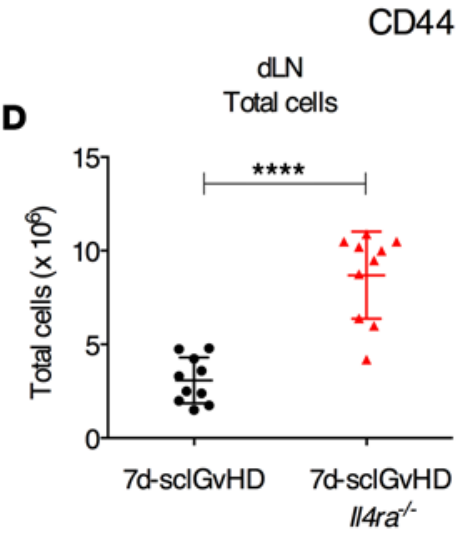

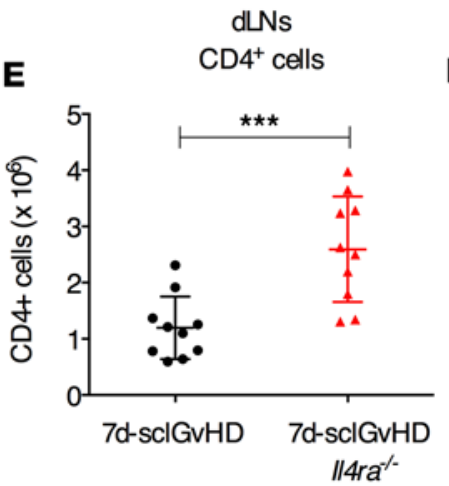

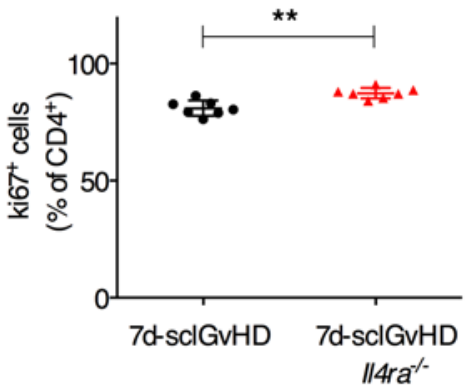

dLNs

Effector-memoryT cells
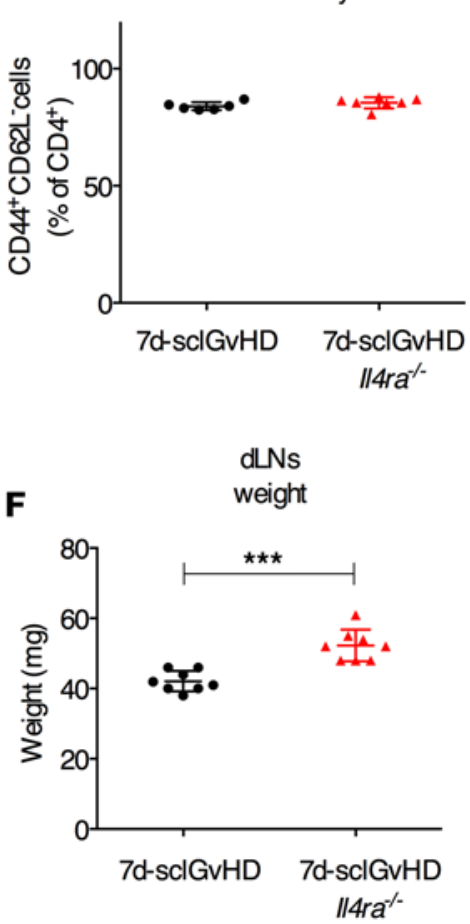

Figure 2. Increased alloreative $\mathrm{CD}^{+} \mathrm{T}$ cells in the dLNs of $7 \mathrm{~d}$-sclGvHD $/ 14 \mathrm{ra}^{-/-}$mice. (A-C) Representative FACS plots and quantification of $\mathrm{CD} 4^{+}$cells expressing

(A) CD69, (B) ki67, or

(C) naive $/ T_{E M}$ markers (CD44 and CD62L) in the dLNs of $7 d$-sclGvHD and 7d-sclGvHD $/ 14 \mathrm{ra}^{-/-}$mice ( $n=6-7$ per group, ${ }^{* *} P<$ 0.01 , Student's $t$ test). (D and $\mathbf{E})$ Absolute number of (D) total cells and (E) $\mathrm{CD}^{+} \mathrm{T}$ cells isolated from the dLNs of 7d-sclGvHD and $7 \mathrm{~d}$-sclCvHD $/ 14 \mathrm{ra}^{-1-}$ mice $(n=10$ per group, ${ }^{* * *} P<0.0001$ and ${ }^{* * *} P$ $<0.001$, Student's $t$ test). (F) dLN weight isolated from 7d-sclGvHD and 7d-sclGvHD $/ 14 \mathrm{ra}^{-1-}$ mice ( $n=8$ per group, ${ }^{* *} P<$ 0.001 , Student's $t$ test). Data are means $\pm S D$. The graphs represent data pooled from 3 independent experiments.

by LECs (19), as well as enzymes that catalyze S1P degradation, including S1P lyase (SGPL1) and the S1P phosphatases (SGPP1, SGPP2) (20-22). qPCR analysis of total dLN tissue revealed that Sphk1 mRNA levels were significantly lower (by about $50 \%$ ) in the dLNs of 7d-sclGvHD Il4ra $^{-1-}$ mice than 7d-sclGvHD controls (Figure 4E). In contrast, there were no differences in expression levels of Sphk2, Spns2, Sgpl1, and Sgpp1 (Supplemental Figure 2, A-D). 

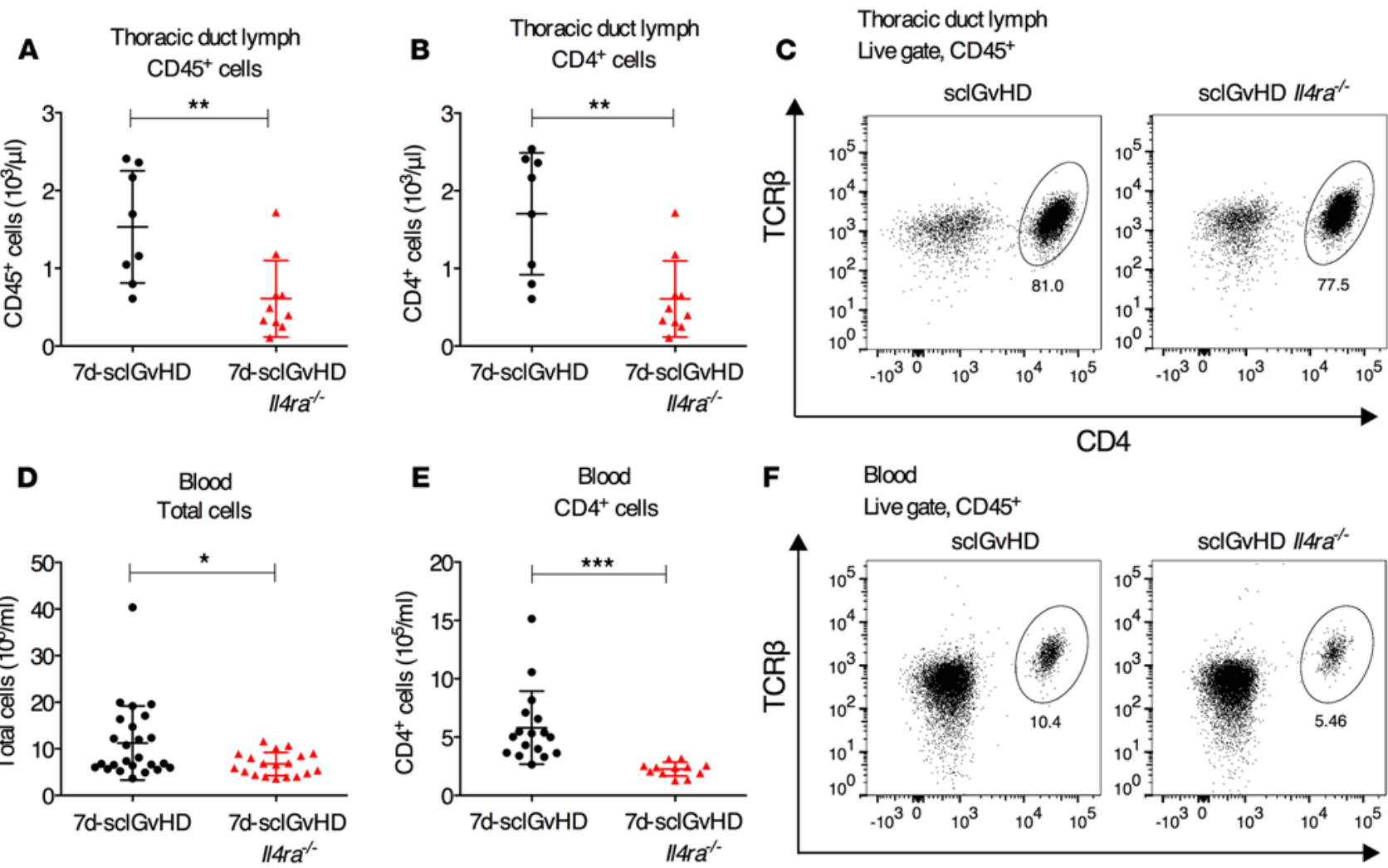

\title{
F $\quad$ Blood
}
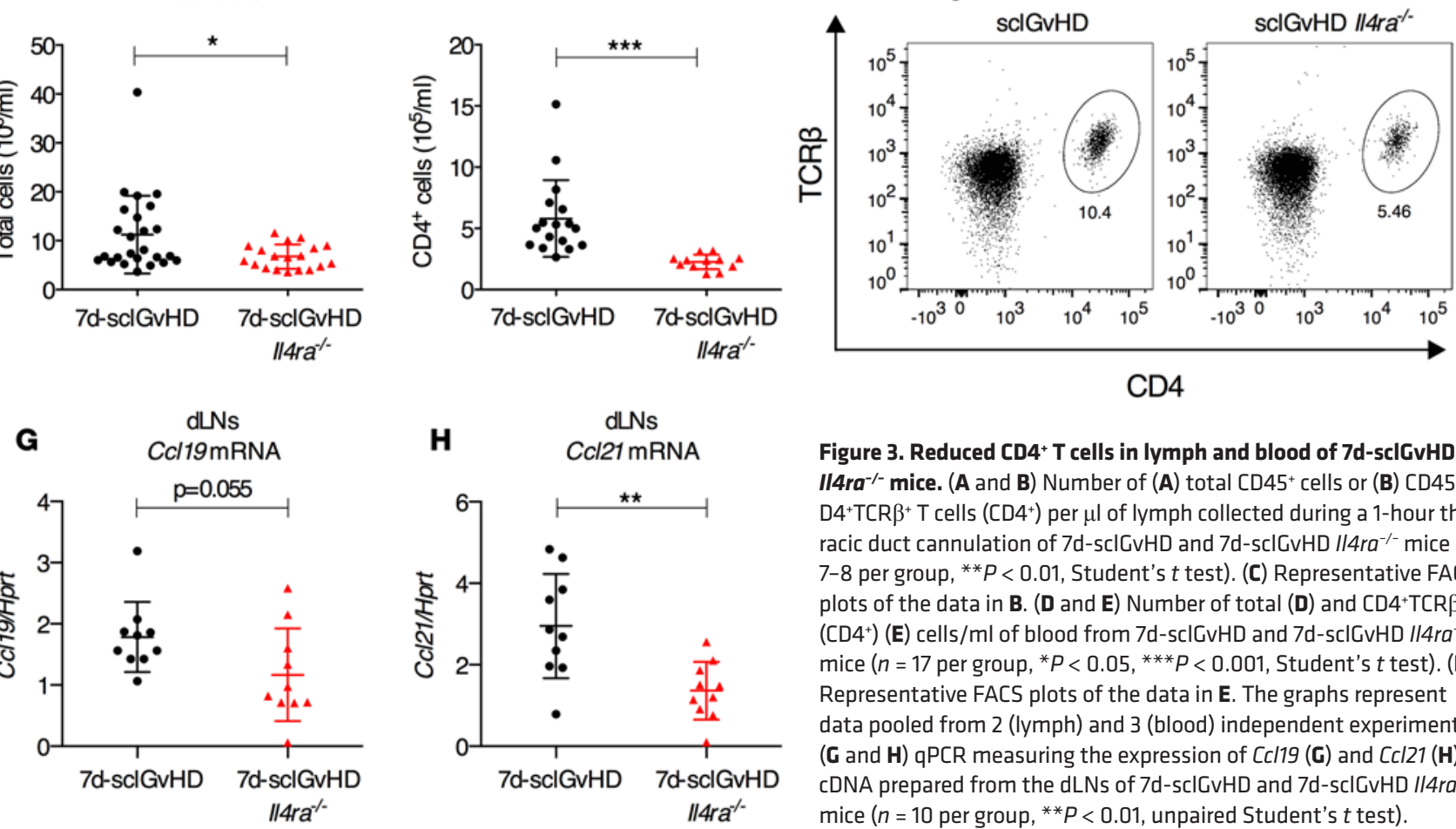

\begin{abstract}
Figure 3. Reduced CD4 ${ }^{+} \mathrm{T}$ cells in lymph and blood of $7 \mathrm{~d}$-sclGvHD II4ra-/- mice. (A and B) Number of (A) total CD45 cells or (B) CD45+CD4 ${ }^{+}$TCR $\beta^{+}$T cells $\left(C D 4^{+}\right)$per $\mu$ l of lymph collected during a 1-hour thoracic duct cannulation of 7d-sclGvHD and 7d-sclGvHD $/ / 4 \mathrm{ra}^{-/-}$mice $(n=$ 7-8 per group, ${ }^{* *} P<0.01$, Student's $t$ test). (C) Representative FACS plots of the data in B. (D and E) Number of total (D) and CD4 $4^{+} T C R \beta^{+} T$ $\left(C^{+}\right)(E)$ cells $/ \mathrm{ml}$ of blood from $7 \mathrm{~d}$-sclCvHD and 7d-sclGvHD $/ 14 \mathrm{ra}^{-/-}$ mice ( $n=17$ per group, ${ }^{*} P<0.05,{ }^{* * *} P<0.001$, Student's $t$ test). (F) Representative FACS plots of the data in $\mathbf{E}$. The graphs represent data pooled from 2 (lymph) and 3 (blood) independent experiments. $(\mathbf{G}$ and $\mathbf{H})$ qPCR measuring the expression of $C c / 19(\mathbf{G})$ and $C c / 21(\mathbf{H})$ in cDNA prepared from the dLNs of 7d-sclGvHD and 7d-sclCvHD $/ 14 \mathrm{ra}^{-/-}$ mice ( $n=10$ per group, ${ }^{* *} P<0.01$, unpaired Student's $t$ test).
\end{abstract}

Sphk1 expression by LECs is reduced in dLNs of Il4ra ${ }^{-1-}$ hosts. We then analyzed the mRNA expression of Sphk1 in sorted cell subsets from dLNs of 7d-sclGvHD and 7d-sclGvHD Il4ra ${ }^{-1-}$ mice. Lymphatic endothelial cells (LECs) and fibroblastic reticular cells (FRCs) were the major populations with detectable amounts of Sphk1 transcript, consistent with previous reports that identified stromal cells as the major source of S1P in naive LNs (17). LECs, but not FRCs, isolated from the dLNs of 7d-sclGvHD Il4ra ${ }^{-1-}$ mice expressed less Sphk1 compared with the same population from 7d-sclGvHD control mice (Figure 5, A-C) while there was no difference in the absolute number of LECs or FRCs recovered from dLNs of both hosts (Figure 5, D and E). Together, these data support the conclusion that the lower S1P concentration in the dLNs of 7d-sclGvHD Il4ra- ${ }^{-1}$ mice is the result of reduced Sphk1 expression by LECs in these mice. Interestingly, LECs isolated from BALB/c Il4ra ${ }^{+/+}$and $\mathrm{Il}_{4 \mathrm{ra}^{-/-}}$mice (i.e., mice without disease) expressed similar amounts of Sphk1 mRNA after short-term in vitro culture. Stimulation with dLN media from 7d-sclGvHD mice resulted in upregulation of Sphk1 expression in Il4ra ${ }^{+/+}$LECs (2- to 6-fold in 5 independent experiments). Sphk1 induction was significantly attenuated in $I l 4 \mathrm{ra}^{-1-}$ LECs, consistent with an intrinsic defect of Sphk1 expression in these cells (Figure 5F). LECs have been shown to express IL4RA $(23,24)$, and stimulation of $I_{44 a^{+/+}}$ LECs with dLN media from 7d-sclGvHD mice enhanced IL4RA expression (Supplemental Figure 3A). 
A

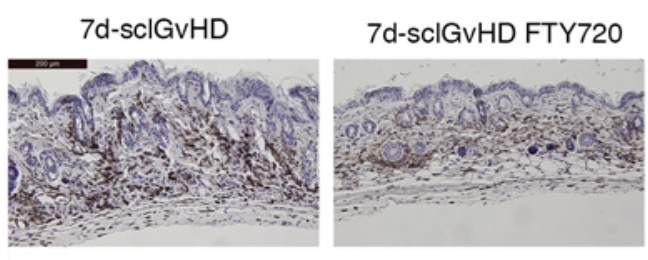

IHC CD3

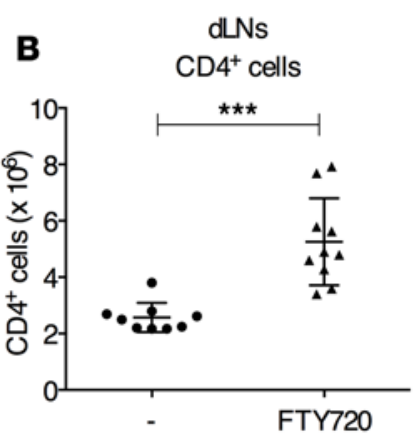

dLNs (CD4+ cells) S1PR1MFI

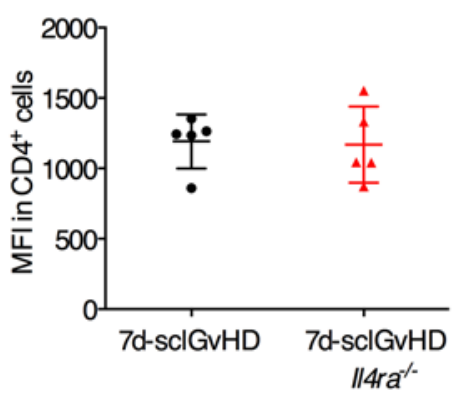

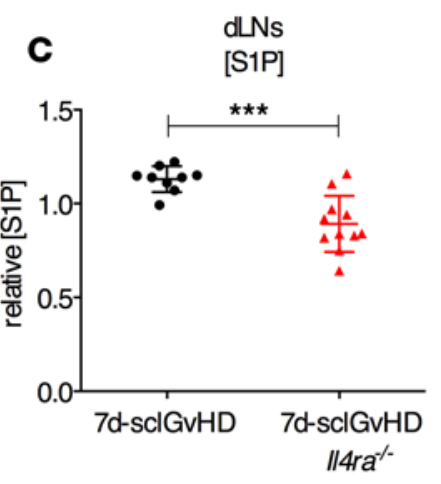

E

dLNs Sphk1 mRNA

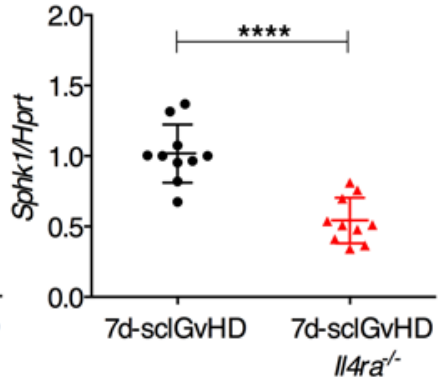

Figure 4. dLNs isolated from 7d-scIGvHD II4ra-/- mice display a defect in S1P pathway. (A and B) Representative IHC of CD3 in back skin sections (A) and number of CD4+ T cells isolated from dLNs (B) of 7d-sclGvHD mice treated with $3 \mathrm{mg} / \mathrm{kg}$ FTY720 or saline for 6 days $\left(n=9-10\right.$ per group, ${ }^{* * *} P<0.001$, Student's $t$ test; 10x. Scale bar: $200 \mu \mathrm{m})$. (C) Relative S1P concentration (S1P) in dLN media prepared from 7d-sclGvHD and 7d-sclGvHD $/ / 4 \mathrm{ra}^{-/-}$mice $(n=9-11$ per group, ${ }^{* *} P<0.001$, Student's $t$ test). (D) Representative plot and MFI for S1PR1 surface staining of CD4+ $T$ cells isolated from dLNs of 7d-sclCvHD and 7d-sclGvHD $/ 14 \mathrm{ra}^{-/-}$mice ( $n=5$ per group). Blood CD4 ${ }^{+}$T cells are used as negative control. (E) qPCR measuring Sphk1 expression in cDNA prepared from the dLNs of 7d-scIGVHD and 7d-sclGvHD $/ 14 \mathrm{ra}^{-/-}$mice $\left(n=10\right.$ per group, ${ }^{* * *} P<0.0001$, Student's $t$ test).

IL13 induced the expression of $C c l 2$, a well-known IL13 target gene (Supplemental Figure 3B) but by itself did not result in upregulated Sphk1 transcription in $\mathrm{IlAra}^{+/+}$LECs (Figure 5F).

Immunofluorescence microscopy for the LEC marker LYVE-1 revealed a collapsed appearance of the lymphatic vessels in dLNs of $7 \mathrm{~d}$-sclGvHD $\mathrm{IlAra}^{-/-}$mice (Figure $5 \mathrm{G}$ ). The aspect ratio as a measure of lymphatic vessel compression (25) was significantly higher in dLN sections from 7d-sclGvHD Il4ra- mice compared with 7d-sclGvHD controls (Figure 5G). Similar changes in lymphatic vessel morphology have been previously described in mice lacking S1P production by LECs (17) or in mice deficient in the S1P transporter SPNS2 (19). Lymphatic vessel morphology therefore supports the conclusion that there is a functionally relevant decrease in tissue S1P levels in the dLNs of 7d-sclGvHD Il4ra ${ }^{-1}$ mice. Correspondingly, we found that mRNA expression of VE-Cadherin, a known target gene of S1P signals in endothelial cells $(17,26-29)$, was significantly lower in 7d-sclGvHD Il4ra- dLNs (Figure 5H).

Secondary transfer of $d L N T$ cells from $7 d$-sclGvHD Il4ra-- mice into blood partially restores disease in Il4ra ${ }^{-1-}$ hosts. If $\mathrm{IlAra}^{-/}$host mice are protected from sclGvHD because the alloreactive T cells are trapped in the dLNs, one would expect that by bypassing this block, one might bring about clinical disease. To test this hypothesis, we isolated $\mathrm{CD}^{+} \mathrm{T}$ cells from dLNs of $7 \mathrm{~d}-\mathrm{sclGvHD} \mathrm{Il4ra}^{-/}$mice (mostly $\mathrm{T}_{\mathrm{EM}}$ cells, Supplemental Figure 4) and i.v. injected them into secondary $7 \mathrm{~d}-\mathrm{sclGvHD} \mathrm{IlAra}^{-1-}$ hosts. An additional group of $7 \mathrm{~d}$-sclGvHD Il4ra ${ }^{-/}$animals received LN cells from B10.D2 mice (mostly naive T cells, $\mathrm{T}_{\text {naive }}$ cells, Supplemental Figure 4) to control for phenotypic effects that were the result of supplying an additional load of alloreactive $\mathrm{T}$ cells.

During the observation period of 6 weeks, 5/15 (33\%) sclGvHD Ill $_{4 a^{-/}}$mice receiving CD4 ${ }^{+} \mathrm{T}$ cells from 7d-sclGvHD $\mathrm{Il4ra}^{-/-}$mice $\left(\mathrm{T}_{\mathrm{EM}} \rightarrow \mathrm{sclGvHD} \mathrm{Il}_{4 \mathrm{ra}}{ }^{-/}\right.$) developed alopecia and thus clinical sclGvHD, compared with 0/12 animals receiving CD4 $4^{+} \mathrm{T}$ cells from B10.D2 donors ( $\mathrm{T}_{\text {naive }} \rightarrow$ sc1-

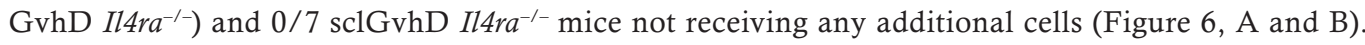
The number of infiltrating $\mathrm{CD}^{+} \mathrm{T}$ cells was much higher in the skin of $\mathrm{T}_{\mathrm{EM}} \rightarrow$ sclGvHD IlAra $^{-/-}$mice 
A

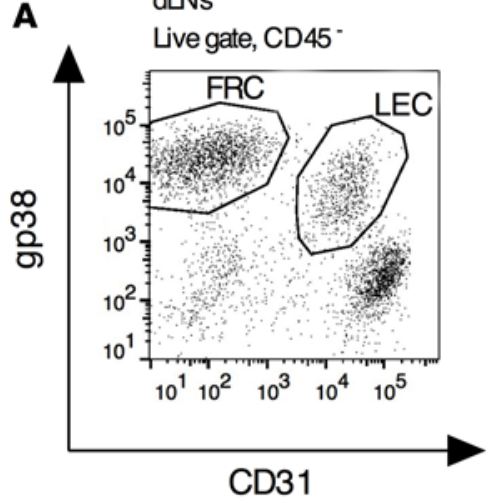

D

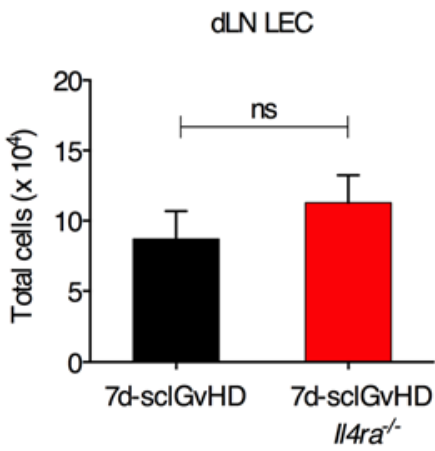

B

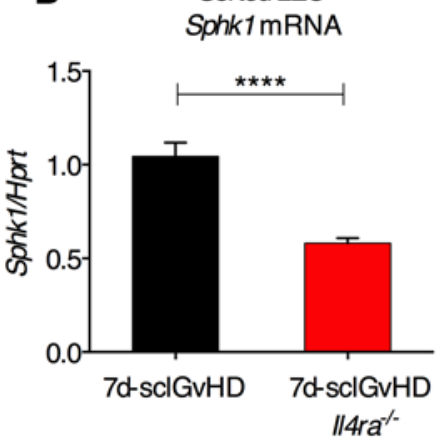

E

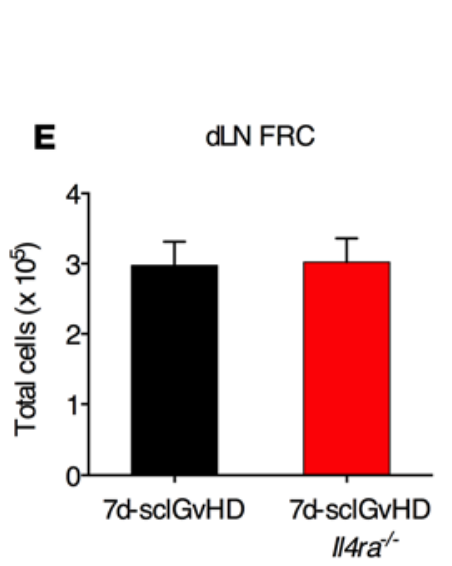

G
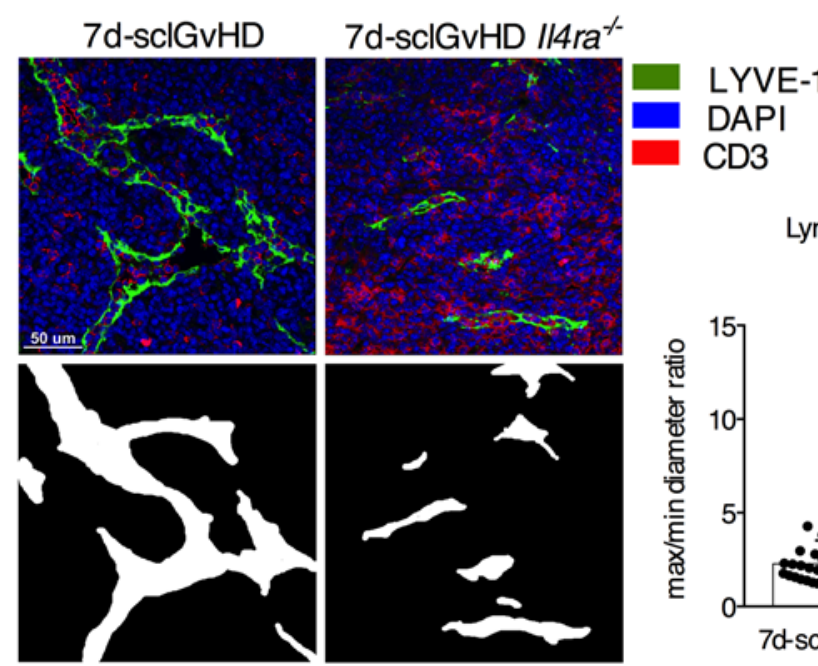

Lymphatics aspect ratio
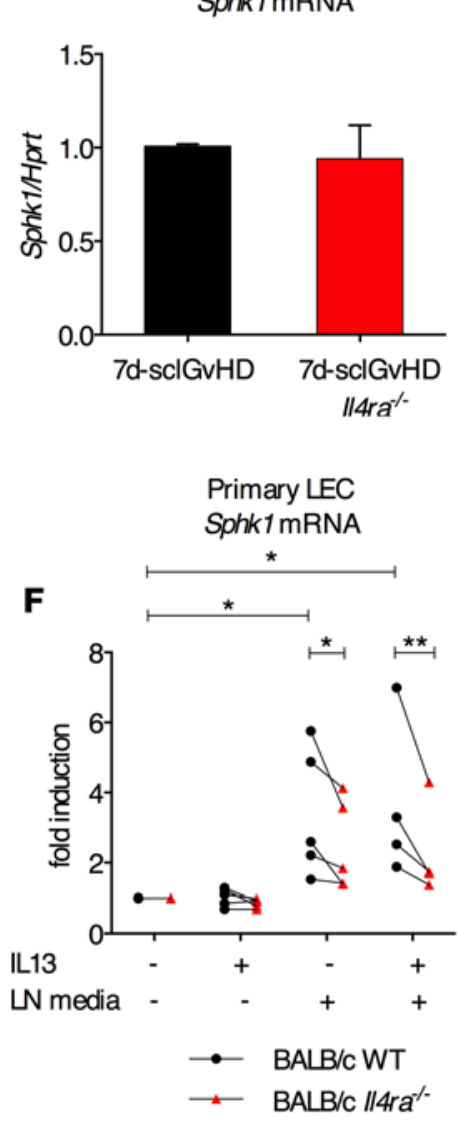

Sphk1 mRNA

Figure 5. dLN LECs express less Sphk1 in 7d-scIGvHD II4ra $\mathbf{r a}^{-/-}$mice. (A) Representative FACS plot indicating the subsets of CD45- $d L N$ cells identified as FRCs (gp38+CD31-) and LECs (gp38+CD31+). (B and C) qPCR measuring Sphk1 expression in (B) LECs or (C) FRCs sorted from dLNs of 7d-sclCvHD and 7d-sclCVHD $/ 14 \mathrm{ra}^{-1-}$ mice ( $n=4$ experiments, with 3-5 mice pooled in each experiment, ${ }^{* * *} P<0.0001$, Student's $t$ test). (D) Absolute number of LECs isolated in B. (E) Absolute number of FRCs isolated in C. (F) qPCR measuring Sphk1 expression in LECs isolated from the dLN of BALB/c WT or $/ 14 \mathrm{ra}^{-/-}$mice and cultured for 2 hours with either IL13 $(20 \mathrm{ng} / \mathrm{ml})$, dLN-media from 7d-sclGvHD (40\%), or a combination of them. Data points represent average of individual experiments. Data points comparing WT or $1 / 4 \mathrm{ra}^{-/-}$LECs from individual experiments are linked by lines. ${ }^{*} P<0.05,{ }^{* *} P<0.01,2$-way ANOVA for repeated measures followed by Bonferroni post-test. The experiments were performed more than 3 times. (G) Frozen sections of inguinal dLNs isolated from $7 \mathrm{~d}$-scl-

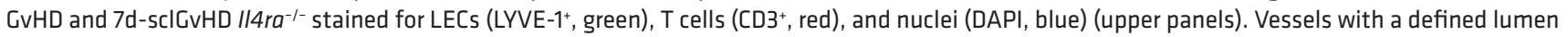
were outlined and the image dichotomized into luminal (white) and nonluminal (black) areas (lower panels). $40 \times$. Scale bar: $50 \mu \mathrm{m}$ ( $n=43-55$ total vessels quantified with Image J Fiji software. The graph on the right represents quantification of the aspect ratios (maximum diameter/minimum diameter) of the lymphatic vessels identified in the left panels. A circular vessel has an aspect ratio of 1. Larger aspect ratios indicate more compressed vessels. $n=5$ mice per group; ${ }^{* *} P<0.001$; Student's $t$ test. (H) qPCR measuring VE-Cadherin expression in cDNA prepared from the dLNs of 7d-sclGvHD and 7d-sclGvHD $1 / 4 \mathrm{ra}^{-/-}$mice ( $n=10$ per group, ${ }^{* *} P<0.0001$, Student's $t$ test). 
A

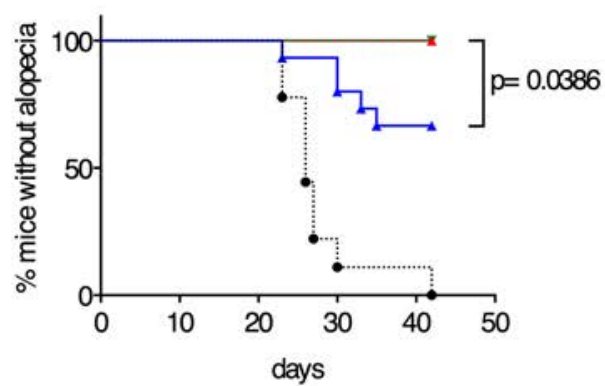

(...... sclGvHD

sclGvHD /14ra ${ }^{-\%}$

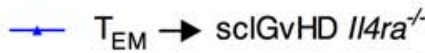

$\rightarrow T_{\text {näive }} \rightarrow$ sclGvHD //4ra ${ }^{\%}$
B

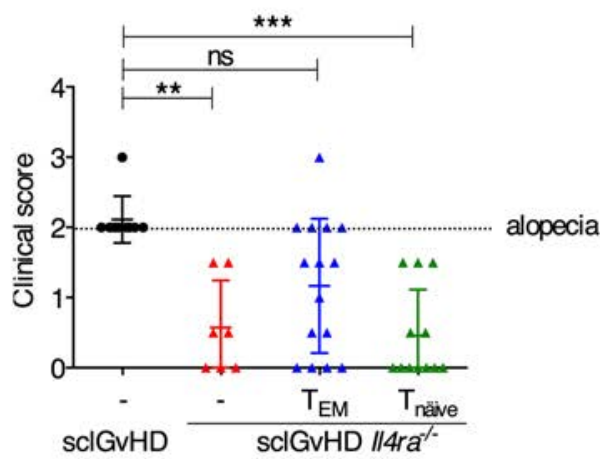

Clinical score

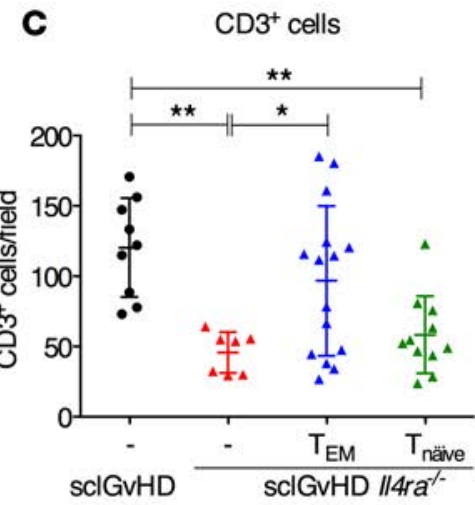

D $\quad$ scIGvHD

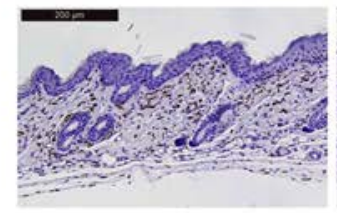

E
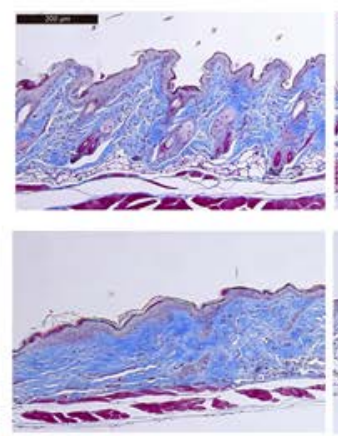

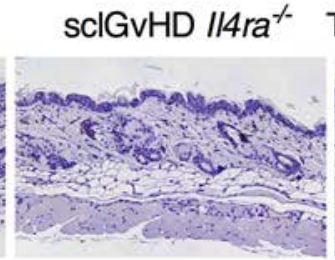

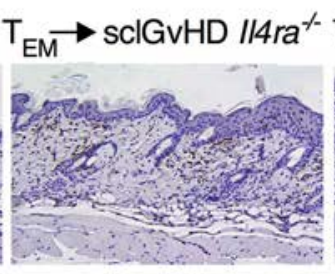

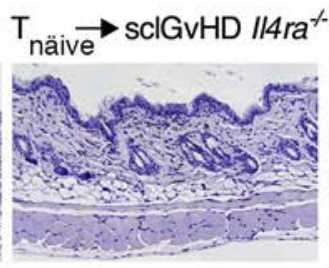

IHC CD3
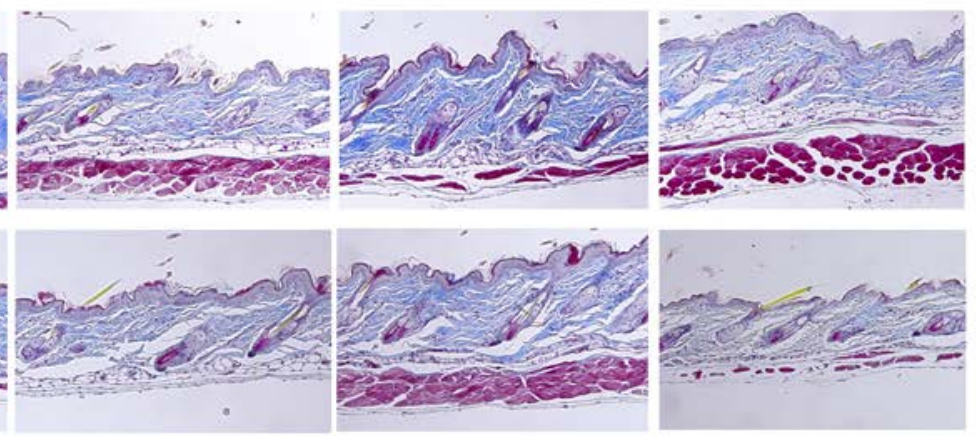

\section{Masson's Trichrome}

Figure 6. Transfer of scIGvHD-activated T cells into the blood of ScIGvHD II4ra-/- mice restores disease. (A-E) Clinical and pathological evaluation of sclGvHD or sclGvHD $/ / 4 \mathrm{ra}^{-/-}$mice, or sclGvHD $/ / 4 \mathrm{ra}^{-/-}$mice that received an additional i.v. injection of CD4+ cells from either B10.D2 mice ( $\mathrm{T}_{\text {naive }}$ cells green)

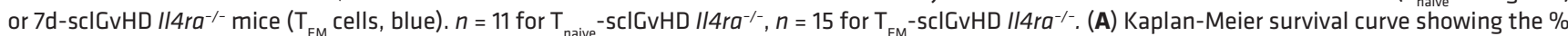
of mice without alopecia (i.e., a clinical score $<2$ ), $P=0.0386$, Mantel-Cox test for survival curves. (B) Clinical scores on day $42,{ }^{* *} P<0.01,{ }^{* *} P<0.001$, one-way ANOVA, Kruskal-Wallis test for comparing ranks. (C and D) IHC staining for CD3 (Scale bar: $200 \mu \mathrm{m}$ ) of histological sections of back skin prepared at sacrifice and cell number quantification, ${ }^{*} P<0.05$, ${ }^{* *} P<0.01$, one-way ANOVA followed by Bonferroni post-test. (E) Masson's Trichrome stain of histological sections of back skin prepared at sacrifice. Lower and upper panel show 2 different mice. Scale bar: $200 \mu \mathrm{m}$.

(irrespective of clinical alopecia) and was statistically indistinguishable from sclGvHD control mice (Figure 6, C and D). Skin sections of $\mathrm{T}_{\mathrm{EM}} \rightarrow \mathrm{sclGvHD} \mathrm{Il}_{\mathrm{H} a^{-/}}$mice with alopecia showed fibrosis similar to sclGvHD mice (Figure 6E), providing additional evidence for the restoration of clinical sclGvHD in the $\mathrm{T}_{\mathrm{EM}} \rightarrow$ sclGvHD Il4ra ${ }^{-1}$ group.

\section{Discussion}

Murine sclGvHD and a subset of SSc patients share a signature of IL13-induced genes in the skin (5, 6, 30). Following up on our published observation that mice deficient for IL4RA, a component of the IL13 receptor, are protected from sclGvHD (5), we demonstrate in this study that the IL13/IL4RA pathway is not only important in the skin, but also plays a critical role during the early phase of the pathogenic immune response in skin dLNs. Specifically, we found that impaired egress of alloreactive $\mathrm{CD} 4^{+} \mathrm{T}$ cells 
from dLNs in IL4RA-deficient mice was associated with a defective SPHK1/S1P pathway in LECs and contributed to the protection of IL4RA-deficient hosts from sclGvHD. This conclusion was supported by restoration of skin disease in a substantial number of $\mathrm{Il4ra}^{-/-}$hosts when $\mathrm{T}_{\mathrm{EM}}$ cells were directly injected into the blood stream.

IL4RA is expressed on multiple cell lineages, including endothelial cells, fibroblasts, and myeloid cells (31-33). The role of IL13 as a local mediator of skin fibrosis in Ssc and other diseases is well established $(34,35)$ and likely involves the activation of dermal fibroblasts and profibrotic macrophages (36). We previously reported that, 2 weeks after splenocyte transfer, the number of $\mathrm{CD} 11 \mathrm{~b}^{+} \mathrm{MHCII}{ }^{+}$dermal macrophages was increased in sclGvHD skin. These host-derived cells expressed IL13, as well as IL4RA, in WT hosts and were significantly less abundant in the skin of $\mathrm{Il4ra}^{-/}$hosts. While the precise role of these cells in the development of skin fibrosis needs further investigation, they represent another potential mechanism

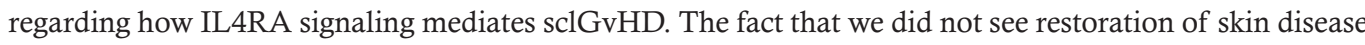
in $100 \%$ of the animals injected with $\mathrm{T}_{\mathrm{EM}}$ cells supports the notion that multiple mechanisms contribute to the protection of IL4RA-deficient hosts from sclGvHD.

Recent studies have shown that LECs express IL4RA and that stimulation with IL13 or IL4 inhibits LEC proliferation $(23,24)$. While we did not observe a difference in the absolute number of LECs in the dLNs of $I l 4 \mathrm{ra}^{+/+}$and $\mathrm{Il}_{4 \mathrm{ra}}{ }^{-/-} \mathrm{d} \mathrm{d}-\mathrm{sclGvHD}$ mice (Figure 5, D and E), our data suggest that the regulation of Sphk1 expression and S1P production is another function of IL4RA in LECs and the collapsed appearance of the lymphatic vessels in $\mathrm{Il4ra}^{-1-}$ hosts (Figure $5 \mathrm{G}$ ) is consistent with a functionally relevant reduction in S1P levels in the dLNs of these mice (Figure 4C). While IL13-dependent induction of S1P has previously been shown in human lung epithelial cells (37), short-term stimulation of $144 \mathrm{ra}^{+/+}$LECs with IL13 upregulated the known IL13 target gene Ccl2 but not Sphk1 (Figure 5F and Supplemental Figure 3B). However, stimulation with cell free extract from d7-sclGvHD mice (LN media) resulted in significantly stronger induction of Sphk1 mRNA in $I_{44 \mathrm{ra}^{+/+}}$LEC than in Il4ra ${ }^{-/-}$LECs (Figure 5F). The induction of Sphk1 mRNA by inflammatory stimuli such as LPS and TGF $\beta$ has been described for other cell types (33, 34). Little is know about the signals that regulate Sphk1 mRNA in LECs inside dLNs. Presumably, the absence or presence of IL4RA signals in LECs modulates Sphk1 mRNA expression and S1P production under particular inflammatory conditions; the molecular link between IL4RA and Sphk1 expression still needs to be explored. To conclusively demonstrate that the lymphocyte egress block in $I l 4 \mathrm{ra}^{-/-}$hosts in the sclGvHD model is mediated specifically via IL4RA deficiency in LECs would require the generation of a host mouse with conditional deletion of Il4ra in LECs. However, the sclGvHD model is highly dependent on the genetic background requiring the combination of a BALB/c host and B10.D2 donor (38). Generation of conditional $\mathrm{KO}$ mouse would thus require a substantial breeding effort to generate LYVE-1-cre Rag2 $2^{-1-}$ mice on a BALB/c background.

Defects in lymphocyte egress from LNs in $\mathrm{Il}_{4 \mathrm{ra}^{-/-}}$mice have not been reported. However, even germline $S p h k 1^{-/-}$mice do not have a strong defect in lymphocyte egress. This is likely due to compensation

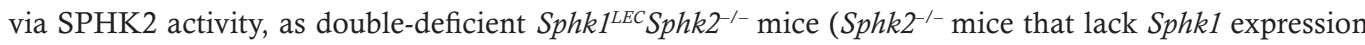
in LECs) exhibit profound lymphopenia in lymph and blood at steady state (17). We speculate that, during sclGvHD, the modest deficiency in SPHK1 observed in Il4ra ${ }^{-/-}$hosts becomes relevant because there is no compensatory upregulation of Sphk2 expression under these conditions (Supplemental Figure 2A). Our data suggest that, in the context of certain inflammatory conditions, even modest changes in the concentration of S1P in the efferent lymphatics may be clinically meaningful. The collapsed morphology of the lymphatics in the dLNs of 7d-sclGvHD $\mathrm{Il}_{4 \mathrm{ra}^{-/}}$mice, which recapitulates the LN pheno-

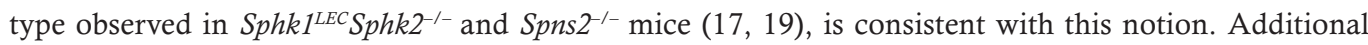
support for the conclusion that the entrapment of T cells in the dLNs of $\mathrm{Il4ra}^{-/-}$hosts is S1P mediated comes from the finding that administration of the S1PR1 superagonist FTY720 to $\mathrm{Il4ra}^{+/+}$hosts resulted in a dLN phenotype similar to $I l 4 \mathrm{ra}^{-/-}$hosts (Figure 4, A and B). Although we did not analyze the effect of FTY720 administration on the clinical sclGvHD phenotype in $I l 4 \mathrm{ra}^{+/+}$hosts, our results are consistent with previous studies demonstrating that long-term treatment of irradiated GvHD mice with FTY720 ameliorated the disease $(39,40)$.

Our study identifies a link between the IL13/IL4RA and S1P pathways in the pathogenesis of sclGvHD. Since drugs targeting IL13, IL4RA, or the S1P pathway have either been approved or are in late development $(20,41-43)$, clinical trials with these agents in patients with the inflammatory form of SSc should be a near term goal. 


\section{Methods}

Mice. Rag2 $2^{-1-} \mathrm{IlAra}^{+/+}$and $\mathrm{Rag}^{-/-} \mathrm{IlAra}^{-/-}$mice on a BALB/c genetic background were generated as described previously (5). B10.D2, BALB/c, and BALB/c Il4ra ${ }^{-/-}$mice were purchased from the Jackson Laboratory and maintained inbred in our facility. All mice were housed in a specific pathogen-free animal facility at the Harvard T.H. Chan School of Public Health, and studies were performed according to institutional and NIH guidelines. The drinking water of all $\mathrm{Rag}^{-1-}$ mice was supplemented with sulfamethoxazole and trimethoprim (Sulfatrim, Hi-Tech Pharmacal, $0.6 \mathrm{mg} / \mathrm{ml}$ drinking solution).

sclGvHD model. Spleens from B10.D2 mice (allogeneic, graft) were gently homogenized, and red blood cells were lysed with BDLyse (BD Biosciences). Approximately $20 \times 10^{6}$ to $30 \times 10^{6}$ cells were injected i.v. into sex-matched 6- to 8-week-old $\mathrm{Rag}^{-1-} \mathrm{IlAra}^{+/+}$and $\mathrm{Rag}^{-/-} \mathrm{Il}_{\mathrm{rra}}{ }^{-/-}$mice to induce GvHD. In long-term experiments, the mice were scored blindly twice weekly for clinical signs of GvHD using a previously described (5) scoring system: 1 = hunched posture and ruffled fur; $2=$ alopecia $<25 \%$ of the body surface; $3=$ alopecia $>25 \%$ of the body surface; $4=$ death or a veterinary order for euthanasia. In the indicated experiments, mice were treated daily for 6 days with $3 \mathrm{mg} / \mathrm{kg}$ FTY720 (Cayman Chemicals) or saline by oral gavage starting on day 1 after splenocyte transfer.

Collection of thoracic duct lymph and blood. Thoracic duct cannulation surgery and collection of lymph was performed as previously described (44). Briefly, mice were administered olive oil by gavage 1 hour prior to the procedure and then anesthetized by i.p. injection of $100 \mathrm{mg} / \mathrm{kg}$ ketamine $\mathrm{HCl}, 10 \mathrm{mg} / \mathrm{kg}$ xylazine, and $3 \mathrm{mg} / \mathrm{kg}$ acepromazine. Mice receive a heparinized polyethylene catheter in the left jugular vein for administration of fluids (Ringer's lactate, $1 \mathrm{U} / \mathrm{ml}$ heparin) prior to and throughout the thoracic duct cannulation procedure. Thoracic duct lymph was collected for 1 hour via a heparinized cannula inserted into the cisterna chyli and assayed by flow cytometry. A peripheral blood sample was collected from the submandibular vein.

Cell isolation, flow cytometry, and sorting. dLNs (2 inguinal, 2 axillary, 2 brachial, 2 popliteal, and 4 cervical LNs per mouse) were isolated and digested as described (45) in RPMI 1640 with 2\% FBS, $0.8 \mathrm{mg} /$ $\mathrm{ml}$ Dispase (Roche Diagnostics), $0.2 \mathrm{mg} / \mathrm{ml}$ Collagenase P (Roche Diagnostics), and $0.1 \mathrm{mg} / \mathrm{ml}$ DNase I (Sigma-Aldrich). Single cell suspensions were stained in the presence of Fc-block with fluorochrome-conjugated antibodies (all purchased from BioLegend unless otherwise indicated) against the following targets: CD4 (RM4-4), CD45 (30-F11), CD3 (1452C11), TCRb (H57-597), CD44 (IM7), CD62L (MEL-14), CD69 (H1.2F3), gp38 (8.1.1), CD31 (MEK13.3), S1PR1 (713412, R\&D Systems). Staining with S1PR1 antibody was performed in the presence of fatty acid-free BSA from Sigma-Aldrich; blood cells were used as S1PR negative controls $(17,46)$. Stained cells were analyzed with the FACS Canto II (BD Biosciences) and FlowJo software. For ki67 (clone 16A8, BioLegend) intracellular staining, cells were fixed and permeabilized with the Foxp3 Fix/Perm Buffer Set (BioLegend). LN stromal cells were sorted as described (45) using a BD FACSAria Fusion cell sorter; $\mathrm{CD} 45^{+}$cells were depleted prior to sorting with anti-biotin magnetic beads (Miltenyi Biotec).

S1P Bioassay. WEHI-S1PR1-Flag-hCD4 B cells were provided by Joao Pereira (Yale University, New Haven, Connecticut, USA) and cultured in RPMI 1640 supplemented with 10\% FBS, 1\% penicillin/ streptomycin, $1 \%$ glutamine, and $0.05 \mathrm{mM} \beta$-Mercaptoethanol. To prepare cell-free dLN media, dLNs were isolated from $7 \mathrm{~d}$-sclGvHD mice, gently homogenized in $300 \mu 1$ of RPMI $+0.5 \%$ fatty acid-free BSA (Sigma-Aldrich) at $4^{\circ} \mathrm{C}$ and centrifuged to remove cells and debris. The S1P bioassay was performed as described (16). WEHI-S1PR1-Flag-hCD4 B cells/ml $\left(2 \times 10^{5}\right)$ were incubated with $100 \%$ dLN media for 40 minutes at $37^{\circ} \mathrm{C}$. S1PR1 surface expression was evaluated on hCD4 ${ }^{+}$cells with anti-S1PR1 antibody followed by a fluorochrome-labeled secondary antibody (Jackson ImmunoResearch). Staining with S1PR1 antibody was performed in the presence of fatty acid-free BSA (Sigma-Aldrich). The relative S1P concentration was obtained from the S1PR1 mean fluorescence intensity (MFI) correcting for dLNs weight with the formula $[\mathrm{S} 1 \mathrm{P}]=1 / \mathrm{MFI} \times 1 / \mathrm{mg} \mathrm{LN}$ weight and normalizing values to the sclGvHD control.

LEC culture. dLN stromal cells were isolated as previously described (45) from BALB/c WT or Il4ra ${ }^{-1}$ mice. Adherent cells derived from digested dLNs were cultured on plates precoated with $0.5 \%$ gelatin (Sigma-Aldrich) in $\alpha$-MEM media with $10 \%$ FBS, $1 \%$ penicillin/streptomycin, and 1\% L-glutamine. After 4 days, the primary culture consisted mainly of FRCs and LECs (45). LECs were separated by positive selection of $\mathrm{CD}_{3} 1^{+}$cells with the EasySep Mouse Biotin selection kit (Stemcell Technologies) and seeded at $1 \times 10^{5} \mathrm{cells} / \mathrm{ml}$ on $0.5 \%$ gelatin precoated plates. LEC purity was assessed by flow cytometry $(70 \%-90 \%$ $\mathrm{CD} 31^{+} \mathrm{gp} 38^{+}$cells). Twenty-four hours later, LECs were starved for 16 hours in $0.5 \%$ FBS media and then 
Table 1. qPCR primer sequence.

\begin{tabular}{|c|c|c|}
\hline Gene & Primer Forward & Primer Reverse \\
\hline Sphk1 $1^{A}$ & ACTCACCGAACGGAAGAACC & AGTCTGGCCGTTCCATTAGC \\
\hline Sgp $/ 7^{A}$ & CGTGGAGTGTCCTGTGTACT & GGCTTTCCTCACCTGTTGTT \\
\hline $\operatorname{Sphk2^{A}}$ & GATGCCСATTGGTGTCCTCC & GATGCССАTTGGTGTССTCС \\
\hline Sgpp $1^{A}$ & CGGGCTGATTCTCATTCCCT & ACAAGAATCCAGCAATGACATCC \\
\hline Spns $2^{A}$ & AACACACGCACATTGATCCT & СTTCACGCTGGAACCTGTGA \\
\hline S1pr1 ${ }^{A}$ & ATGGTGTCCACTAGCATCCC & CGATGTTCAACTTGCCTGTGTAG \\
\hline$\| 4 r a^{B}$ & TCTGCATCCCGTTGTTTTGC & GCACCTGTGCATCCTGAATG \\
\hline $\mathrm{CCl} 2^{\mathrm{B}}$ & GGCTCAGCCAGATGCAGTTAA & ССTACTCATTCGGATCATCTTCCT \\
\hline Ccl19A & CCTGGGAACATCGTGAAAGC & TAGTGTGGTGAACACAACAGC \\
\hline$C c / 21^{A}$ & АСTCTGAGССTССTTAGССT & TAAGGCAGCAGTCCTGACCC \\
\hline$V E-C a d^{A}$ & CACGGACAAGATCACСTCCT & GGTAGCATGTTGGGGGTGTC \\
\hline$H_{p r t}{ }^{B}$ & GTTAAGCAGTACAGCCCCAAA & AGGGCATATCCAACAACAAACTT \\
\hline
\end{tabular}

stimulated with IL13 (20 ng/ml, R\&D Systems), cell-free dLN media, prepared as for the S1P bioassay (40\%) or both for 2 hours.

$R N A$ isolation, $q P C R$. RNA was extracted from whole dLNs with Trizol reagent (Qiagen). RNA from sorted cells and primary LECs was isolated with the RNeasy Micro kit (Qiagen) following the manufacturer's instructions. RNA was reverse transcribed with the Affinity Script qPCR CDNA Synthesis Kit (Agilent Technology). qPCR was performed using Sybr green reagent (Invitrogen). Primers are listed in Table 1. Data were normalized to Hprt.

Histology, IHC, and immunofluorescence. Back skin was isolated from sclGvHD mice 7 or 42 days after splenocyte transfer, fixed in 10\% formalin, and embedded in paraffin. Two H\&E-stained back skin tissue samples per mouse were scored blindly by an observer experienced in SSc pathology (R. Lafyatis) for inflammation, using a semiquantitative scale from $0-3$. T cell IHC on paraffin-embedded skin sections was performed using anti-CD3 antibody (A0452, DAKO) following manufacturer's instructions. The number of infiltrating $\mathrm{CD}^{+}$cells was evaluated blindly on 4 representative $20 \times \mathrm{IHC}$ pictures with Image J software. Collagen was stained with the Masson's Trichrome staining kit (Polysciences). Frozen sections of skin dLNs were prepared from sucrose saturated, paraformaldhehyde fixed tissue embedded in Optimal Cutting Temperature medium. Frozen sections $(10 \mu \mathrm{m})$ were stained with DAPI, (Sigma-Aldrich) FITC-conjugated LYVE-1 antibody (ALY7, eBioscience), and AlexaFluor 594-conjugated CD3 antibody (17A2, BioLegend). Sections were imaged using a Leica SP5X laser-scanning confocal microscope. To determine compression of lymphatic vessels, the images were analyzed with ImageJ/Fiji software, and the aspect ratio was determined for all vessels with a defined lumen by calculating the ratio of maximum to minimum diameters as in ref. 25. An aspect ratio of 1 is a perfectly round vessel, and higher numbers indicate more compressed vessels.

Adoptive transfer of naive or activated $T_{E M}$ cells into $7 d$-sclGvHD Il4ra ${ }^{-1-}$ mice. $\mathrm{CD}^{+}$cells were isolated from dLNs of B10.D2 mice or 7d-sclGvHD Il4ra $^{-1-}$ mice by negative selection with the CD4 ${ }^{+} \mathrm{T}$ cells isolation II kit (Miltenyi Biotec). Purity of the cells was assessed by flow cytometry $\left(88 \%-95 \%\right.$ CD $\left.4^{+}\right)$. Purified CD $4^{+}$ cells $\left(2 \times 10^{6}\right.$ to $\left.3 \times 10^{6}\right)$ were injected i.v. into 7d-sclGvHD $\mathrm{Ilr}_{4 \mathrm{ra}^{-1}}$ mice. Mice were blindly monitored for clinical signs of GvHD as described and sacrificed on day 42 for histological analysis of the skin.

Statistics. The number of animals to be used in each experiment was estimated using the G*Power 3.1 program, considering a statistical power of $80 \%$ and a significance level of 0.05 . Experiments with 2 groups were analyzed with unpaired Student's $t$ tests for continuous variables, and Mann-Whitney $U$ tests were used for categorical values. Two-way ANOVA followed by Bonferroni post-test correction for multiple hypothesis testing was used for experiments with multiple groups. A significance level $\alpha=0.05$ was considered significant. Log-rank (Mantel-Cox) test for Kaplan-Meier survival curves was used for the clinical score in Figure 6 , and as we compared 2 of the 4 groups, the significance level was adjusted to $\alpha=0.025$. All statistical analyses were performed with GraphPad Prism software. Error bars represent \pm SD of biological replicates. All experiments were performed at least 3 times unless otherwise indicated. 
Study approval. The Institutional Animal Care and Use Committee at Harvard Medical School (IACUC protocol 04911) approved this study.

\section{Author contributions}

KU designed, performed and analyzed the majority of the experiments. DA, VC, AG, KT, RL, UHVA, JE, and AOA contributed with ideas and experimental support. AOA supervised the design and interpretation of the results. KU, JE, and AOA wrote the manuscript with the help of DA, VC, AG, KT, RL and UHVA.

\section{Acknowledgments}

This work was supported by grants from the Scleroderma Foundation and Burroughs Wellcome Fund (to AOA), US National Institutes of Health (P01 AI045757 and R21 CA182598 to VC), and the Cancer Research Institute (to VC). This work was also partly supported by the US NIH grants P01 AI112521 and R01 AI111595 (to UHVA); 5T32 HL066987 (to DA); and R03 AR066357-01A1 (to JE). Additional support to UHVA was provided by U19AI095261 and the Ragon Institute of MGH, MIT, and Harvard. This work was also supported by NIH, National Institute of Arthritis Musculoskeletal and Skin Disease grants: 5P30AR061271 and 2R01AR051089 to RL. We thank Joao Pereira for his helpful discussions and for providing the WEHI-S1PR1-Flag-hCD4 B cell line.

Address correspondence to: Antonios O. Aliprantis, Merck Research Laboratories, 33 Avenue Louis Pasteur, Boston, Massachusetts 02115, USA. Phone: 617.992.3040.E-mail: antonios.aliprantis@gmail.com or aaliprantis@partners.org.

1. Elhai M, Meune C, Avouac J, Kahan A, Allanore Y. Trends in mortality in patients with systemic sclerosis over 40 years: a systematic review and meta-analysis of cohort studies. Rheumatology (Oxford). 2012;51(6):1017-1026.

2. Allanore Y, Distler O. Systemic sclerosis in 2014: Advances in cohort enrichment shape future of trial design. Nat Rev Rheumatol. 2015;11(2):72-74.

3. Milano A, et al. Molecular subsets in the gene expression signatures of scleroderma skin. PLoS One. 2008;3(7):e2696.

4. Ruzek MC, Jha S, Ledbetter S, Richards SM, Garman RD. A modified model of graft-versus-host-induced systemic sclerosis (scleroderma) exhibits all major aspects of the human disease. Arthritis Rheum. 2004;50(4):1319-1331.

5. Greenblatt MB, et al. Interspecies comparison of human and murine scleroderma reveals IL-13 and CCL2 as disease subset-specific targets. Am J Pathol. 2012;180(3):1080-1094.

6. Sargent JL, et al. Interspecies comparative genomic identifies optimal mouse models of systemic sclerosis. [published online ahead of print March 24, 2016]. Arthritis Rheumatol. doi: 10.1002/art.39658.

7. Wynn TA. Type 2 cytokines: mechanisms and therapeutic strategies. Nat Rev Immunol. 2015;15(5):271-282.

8. LaPorte SL, et al. Molecular and structural basis of cytokine receptor pleiotropy in the interleukin-4/13 system. Cell. 2008;132(2):259-272.

9. Malhotra D, et al. Transcriptional profiling of stroma from inflamed and resting lymph nodes defines immunological hallmarks. Nat Immunol. 2012;13(5):499-510.

10. Wysocki CA, Panoskaltsis-Mortari A, Blazar BR, Serody JS. Leukocyte migration and graft-versus-host disease. Blood. 2005;105(11):4191-4199.

11. Masopust D, Schenkel JM. The integration of T cell migration, differentiation and function. Nat Rev Immunol. 2013;13(5):309-320.

12. Förster R, Davalos-Misslitz AC, Rot A. CCR7 and its ligands: balancing immunity and tolerance. Nat Rev Immunol. 2008;8(5):362-371.

13. Pham TH, Okada T, Matloubian M, Lo CG, Cyster JG. S1P1 receptor signaling overrides retention mediated by G alpha i-coupled receptors to promote T cell egress. Immunity. 2008;28(1):122-133.

14. Cyster JG, Schwab SR. Sphingosine-1-phosphate and lymphocyte egress from lymphoid organs. Annu Rev Immunol. 2012;30:69-94.

15. Mandala S, et al. Alteration of lymphocyte trafficking by sphingosine-1-phosphate receptor agonists. Science. 2002;296(5566):346-349.

16. Pappu R, et al. Promotion of lymphocyte egress into blood and lymph by distinct sources of sphingosine-1-phosphate. Science. 2007;316(5822):295-298.

17. Pham TH, et al. Lymphatic endothelial cell sphingosine kinase activity is required for lymphocyte egress and lymphatic patterning. J Exp Med. 2010;207(1):17-27.

18. Spiegel S, Milstien S. The outs and the ins of sphingosine-1-phosphate in immunity. Nat Rev Immunol. 2011;11(6):403-415.

19. Nagahashi M, et al. Spns2, a transporter of phosphorylated sphingoid bases, regulates their blood and lymph levels, and the lymphatic network. FASEB J. 2013;27(3):1001-1011.

20. Kunkel GT, Maceyka M, Milstien S, Spiegel S. Targeting the sphingosine-1-phosphate axis in cancer, inflammation and beyond. Nat Rev Drug Discov. 2013;12(9):688-702.

21. Peest U, Sensken SC, Andréani P, Hänel P, Van Veldhoven PP, Gräler MH. S1P-lyase independent clearance of extracellular sphingosine 1-phosphate after dephosphorylation and cellular uptake. J Cell Biochem. 2008;104(3):756-772.

22. Schwab SR, Pereira JP, Matloubian M, Xu Y, Huang Y, Cyster JG. Lymphocyte sequestration through S1P lyase inhibition and disruption of S1P gradients. Science. 2005;309(5741):1735-1739. 
23. Savetsky IL, et al. Th2 cytokines inhibit lymphangiogenesis. PLoS One. 2015;10(6):e0126908

24. Shin K, et al. TH2 cells and their cytokines regulate formation and function of lymphatic vessels. Nat Commun. 2015;6:6196.

25. Padera TP, Stoll BR, Tooredman JB, Capen D, di Tomaso E, Jain RK. Pathology: cancer cells compress intratumour vessels. Nature. 2004;427(6976):695

26. Gaengel K, et al. The sphingosine-1-phosphate receptor S1PR1 restricts sprouting angiogenesis by regulating the interplay between VE-cadherin and VEGFR2. Dev Cell. 2012;23(3):587-599.

27. Krump-Konvalinkova V, et al. Stable knock-down of the sphingosine 1-phosphate receptor S1P1 influences multiple functions of human endothelial cells. Arterioscler Thromb Vasc Biol. 2005;25(3):546-552.

28. Lee MJ, et al. Vascular endothelial cell adherens junction assembly and morphogenesis induced by sphingosine-1-phosphate. Cell. 1999;99(3):301-312.

29. Pfeiffer F, et al. Distinct molecular composition of blood and lymphatic vascular endothelial cell junctions establishes specific functional barriers within the peripheral lymph node. Eur J Immunol. 2008;38(8):2142-2155.

30. Wynn TA, Ramalingam TR. Mechanisms of fibrosis: therapeutic translation for fibrotic disease. Nat Med. 2012;18(7):1028-1040.

31. Fertin C, Nicolas JF, Gillery P, Kalis B, Banchereau J, Maquart FX. Interleukin-4 stimulates collagen synthesis by normal and scleroderma fibroblasts in dermal equivalents. Cell Mol Biol. 1991;37(8):823-829.

32. Gordon S. Alternative activation of macrophages. Nat Rev Immunol. 2003;3(1):23-35.

33. Lowenthal JW, et al. Expression of high affinity receptors for murine interleukin 4 (BSF-1) on hemopoietic and nonhemopoietic cells. J Immunol. 1988;140(2):456-464.

34. Fuschiotti P, Larregina AT, Ho J, Feghali-Bostwick C, Medsger TA. Interleukin-13-producing CD8+ T cells mediate dermal fibrosis in patients with systemic sclerosis. Arthritis Rheum. 2013;65(1):236-246.

35. Oh MH, et al. IL-13 induces skin fibrosis in atopic dermatitis by thymic stromal lymphopoietin. J Immunol. 2011;186(12):7232-7242.

36. Wynn TA. Common and unique mechanisms regulate fibrosis in various fibroproliferative diseases. J Clin Invest. 2007;117(3):524-529.

37. Kono Y, et al. Sphingosine kinase 1 regulates mucin production via ERK phosphorylation. Pulm Pharmacol Ther. 2010;23(1):36-42

38. Stenger EO, Turnquist HR, Mapara MY, Thomson AW. Dendritic cells and regulation of graft-versus-host disease and graft-versus-leukemia activity. Blood. 2012;119(22):5088-5103.

39. Huu DL, et al. FTY720 ameliorates murine sclerodermatous chronic graft-versus-host disease by promoting expansion of splenic regulatory cells and inhibiting immune cell infiltration into skin. Arthritis Rheum. 2013;65(6):1624-1635.

40. Taylor PA, et al. Insights into the mechanism of FTY720 and compatibility with regulatory T cells for the inhibition of graftversus-host disease (GVHD). Blood. 2007;110(9):3480-3488.

41. Bankaitis KV, Fingleton B. Targeting IL4/IL4R for the treatment of epithelial cancer metastasis. Clin Exp Metastasis. 2015;32(8):847-856

42. Kopf M, Bachmann MF, Marsland BJ. Averting inflammation by targeting the cytokine environment. Nat Rev Drug Discov. 2010;9(9):703-718.

43. Plano D, Amin S, Sharma AK. Importance of sphingosine kinase (SphK) as a target in developing cancer therapeutics and recent developments in the synthesis of novel SphK inhibitors. J Med Chem. 2014;57(13):5509-5524.

44. Massberg S, et al. Immunosurveillance by hematopoietic progenitor cells trafficking through blood, lymph, and peripheral tissues. Cell. 2007;131(5):994-1008.

45. Fletcher AL, et al. Reproducible isolation of lymph node stromal cells reveals site-dependent differences in fibroblastic reticular cells. Front Immunol. 2011;2:35.

46. Lo CG, Xu Y, Proia RL, Cyster JG. Cyclical modulation of sphingosine-1-phosphate receptor 1 surface expression during lymphocyte recirculation and relationship to lymphoid organ transit. J Exp Med. 2005;201(2):291-301. 